maternal age was a significant risk factor for primiparous patients. For both groups, MROP was associated with greater blood loss.

Conclusion These results show an increase in the rate of MROP as compared to earlier studies and identifies similar risk factors. Of note, maternal age in primiparous women and use of oxytocin are factors that have undergone change since earlier studies and may be contributing to the rise in the rates of MROP in the industrialised world.

\section{PL.73 DOES WHO WALKS INTO THEATRE MAKE A DIFFERENCE FOR SECOND STAGE DELIVERIES AND SHOULD IT?}

doi:10.1136/archdischild-2013-303966.255

HR Simpson. James Cook University Hospital, Middlesbrough, UK

Detailed statistics on individual acchouchers have been kept from 2009 at JCUH. This paper is based on that data. It considers the influence of individual acchouchers on the success rate of second stage delivery in theatre.

Consultant attendance is currently $92 \%$. Before the introduction of 98 hour cover attendance was $74 \%$.

There was considerable variation in the success rates of individual achouchers from $20-78 \%$. If instrumentation was attempted then rates varied from $40-87 \%$.

Complication rates of the successful trials were compared. The consultants were split into three groups:

Success rate of $\leq 40 \%$

Success rate of $41-70 \%$

Success rate of $>70 \%$

The least maternal complications (third/fourth degree tear and $\mathrm{PPH}>1.5 \mathrm{l}$ ) occurred in the group with the highest success rate.

The number of neonatal complications (NNU admission of a baby $>37$ weeks, apgar $<7$ at 5 mins, cord $\mathrm{ph}<7.1$ and shoulder dystocia) were very small but they did not increase in the group with the higher delivery rate.

Conclusions there is considerable variation in success rates in women taken to theatre in the second stage dependant on the acchoucher. If we are to lower the caesarean section rate we need to look at the skills of individuals. A higher success rate does not appear to be associated with a higher complication rate. Further assessment is required looking at the complications of caesareans in the second stage. Data collection has commenced in 2012 to try and look at this further.

\section{PL.74 INDUCTION OF LABOUR WITH PROPESS ${ }^{\circledR}$}

doi:10.1136/archdischild-2013-303966.256

M Isdale, J Danson-Smith, J Amu. Blackpool Teaching Hospital NHS Foundation Trust, Blackpool, UK

Introduction Propess ${ }^{\circledR}$ is a vaginal delivery system that consists of a non-biodegradable polymeric drug delivery device containing $10 \mathrm{mg}$ dinoprostone (Prostaglandin $\mathrm{E}_{2}$ ) dispersed throughout its matrix. Introduction in most units as a method of induction of labour is largely due to reported benefits which include effectiveness and better patient experience.

Objective To evaluate the use of Propess ${ }^{\circledR}$ for induction of labour and the pregnancy outcome of the recipients.

Method A retrospective review of all induction of labour undertaken with Propess ${ }^{\circledR}$ over 4 months. Data was extracted from Euro king database unto excel for analysis.

Result 66 patients, with parity not more than 3, were induced with Propess ${ }^{\circledR}$ at term. The commonest indication for induction was post maturity $(74 \%)$ however $4(6 \%)$ high risk patients were induced with Propess ${ }^{\circledR} .93 \%$ of patients who achieved spontaneous vaginal delivery received single dose Propess ${ }^{\circledR}$. Patients delivered through caesarean section had slightly bigger babies (SVD $3.666 \mathrm{~kg}$ CS $3.713 \mathrm{~kg}$ ), were more likely to receive additional agents (prostin) to ripen the cervix and syntocinon to augment labour. Overall 55 patients (83\%) achieved vaginal birth (spontaneous and assisted) and $17 \%$ via emergency caesarean section.

Conclusion Propess ${ }^{\circledR}$ is a cost effective agent for induction of labour as over $80 \%$ of patients achieved vaginal birth after single dose. However further studies are required to determine choice and/ or dosage of additional cervical ripening agents suitable for use when single dose Propess ${ }^{\circledR}$ fails. This will help to reduce the number of caesarean sections performed for 'failed induction' after single dose Propess ${ }^{\circledR}$.

\section{PL.75 AN AUDIT OF LOWER URINARY TRACT INJURIES IN A LARGE OBSTETRIC UNIT - 2 YEAR OUTCOME}

doi:10.1136/archdischild-2013-303966.257

BD Upadhyay. St George's University London, London, UK

Objective The main objective of this audit was to investigate the number of bladder injuries after delivery and compare it to the existent incidence in literature.

Method Retrospective medical record review of patients in Kingston Hospital's Obstetric department who had suffered from either ureteric or bladder injury between January 2011 and November 2012. A record of all these patients was recovered from the Obstetric electronic database.

Results There were a total of 11,246 deliveries between January 2011 and November 2012. Out of these 6,356 were normal vaginal deliveries, 1,684 were instrumental deliveries and 3,206 were caesarean sections. There were 6 cases of bladder injury found, all during caesarean section. 3 out of the 6 cases had caesarean section before the index pregnancy. 3 others had placenta praevia. Of these 3 previous caesarean section patients, 2 were unsuccessful at vaginal birth after caesarean section. One of them had a scar dehiscence extending to the bladder. Subsequent repair in all 6 cases was successful as indicated by normal imaging and a successful trial without catheter following 2 weeks of being catheterised.

Conclusion The rate of bladder injury found was $6 / 11,246$ deliveries between January 2011 and November 2012. The incidence of bladder injury following caesarean delivery, 6/3,206 is comparable to the literature. This study also found that having a previous caesarean delivery increased the likelihood of having a bladder injury after caesarean delivery. Subsequent repair of bladder injury demonstrated good success rates.

\section{PL.76 FAILURE TO IDENTIFY THE NEW ONSET OF INTRAPARTUM RISK FACTORS IN LOW RISK PREGNANCIES: A MISSED OPPORTUNITY FOR BETTER NEONATAL OUTCOME}

doi:10.1136/archdischild-2013-303966.258

'NM Doherty, ' $\mathrm{K}$ Connor, ' 'G Krystowski, 1,2J Costa. 'Royal Jubilee Maternity Hospital, Belfast, UK; ${ }^{2}$ Queens University, Belfast, UK

Objective Evaluate the risk factors and immediate outcome of term neonates born with Apgar Score (AS) of $<7$ at 5 minutes.

Methods Retrospective review of maternity database to identify 187 live born neonates with AS $<7 @ 5$ minutes, during a 2 year period, in a large tertiary care unit in N.Ireland. Excluding multiple pregnancies and neonates born preterm or with congenital malformations, a sample of 57 term neonates were identified for analysis of case notes.

Results $63 \%$ of mothers were primigravida, at 37 weeks or more, with $54 \%$ of them being forty weeks or more at delivery. Only $28 \%$ of pregnancies were known to be high risk at the onset of labour 